\title{
An XML Approach to Student Usage Reflection in Online Courses
}

\author{
K O Chow, Alan Y K Chan \\ Department of Computer Science \\ City University of Hong Kong
}

\author{
K S Cheung \\ School of Continuing Education \\ Hong Kong Baptist University
}

\begin{abstract}
Online course is common in the education scene. Students use online course for a variety of reasons. However, their usage, as recapped in log, is worthy of further investigation. This article proposes an XML web log data model to represent web server log files in online courses. The implementation of the XML web log data model and how it is used to reflect student usage are discussed. Using XML and its related technologies such as XSLT to represent and process log data is a flexible and extensible method to visualize and compare student usage. Student would be able to recap and compare his/her usage with others and improve in using the online courses.
\end{abstract}

\section{Introduction}

Online courses have been widely used to support teaching and learning in higher education. It is essential to develop methods to properly assess student usage in online courses. One common approach is to use existing web log analysis tools to process server $\log$ files. Such tools are easy to setup, user-friendly and provide customizable template-based reports. Although they are powerful in term of functionalities, the reports are very general and focus only on the web server traffic. They are difficult to interpret due to the dynamic structure and content of the website and do not truly reflect student usage in online courses.

Generating user sessions and categorizing URLs from the server log files can enhance the data and provide better analysis in student usage. This paper proposes the use of XML to model the web log data. There are several benefits with this XML approach. For example, individual access history can be revealed, reflecting student usage in online course. The XML data can be easily integrated into online courses, which are delivered on third parties learning management system (LMS) such as WebCT.

The next section discusses the characteristics and the functions of online courses. Section 3 discusses existing web $\log$ analysis technologies and their deficiency. Section 4 describes the implementation of the XML log data model. Section 5 presents the result and how it is used to reflect student usage. Finally, evaluation of the work and possible improvements are discussed in the conclusion.

\section{Student Usage in Online Courses}

To understand and define student usage in online courses, it is essential to identify the characteristics and functions of online courses. These are discussed in the following sub-sections.

\subsection{Characteristics of online courses}

Online courses provide a flexible learning environment where both instructors and students can teach and learn regardless of time and location. The learning process can be self-paced, independent, collaborative and continuous in online courses. Online courses support high quality learning by offering different kinds of environments such as synchronous or asynchronous or both. Some environments and approaches facilitates student learning while others impede it [1]. A traditional classroom provides a faceto-face learning environment where instructors can direct and take active immediate role in class at a fixed time and place. Online courses provide alternative opportunities for current on-campus students to take 
classes that they could not take otherwise due to time conflicts with other courses or work [2]. In traditional classroom, it is often instructional where the instructors transfer the knowledge directly to the students. It does not reflect the students' understanding of the knowledge effectively. However, the dynamic nature in the online courses supports better communication such as self-reflections and peer-to-peer reviews. This change in communication transforms the learning process where knowledge is constructed actively by cognition [3].

\subsection{Functions of online courses}

Learning management system (LMS) is commonly used as a platform for the delivery of online courses. LMS is defined as a distinct, pedagogically meaningful and comprehensive system by which learners and faculty can participate in the learning and instructional process at anytime and any place [4]. Its functions can be categorized into several components such as content delivery (organizer and content pages, URLs, etc), communication and collaboration (chat, whiteboard, forum, mail, calendar, etc), assessment (quizzes, assignments, self-test, etc) and class administration (grade book, syllabus, etc) [5].

\subsection{Student usage}

In online courses, student usage activities include accessing course materials, posting and reading discussion forums, taking online quizzes, interacting synchronously in chat room and white board, sharing resources, etc. They are usually stored in either databases or data files and their transaction log are recorded automatically in web access log files in a specific format. Analysis of these databases (forums, quizzes, etc), data files (chat, shared resources, etc) and access log files (page visits) can be used to reflect student usage in online courses. Ideally, it is better to use all the available data sources for reflecting student usage. However, use of databases and data files can be difficult and requires extensive understanding of the data structure and format in the LMS platform. Access $\log$ files are more feasible due to their large size and standard format. It can be based primarily on usage such as access frequency, duration and page views of the requested resources. It can also be further analyzed based on a time series since online courses are continuous. Generating user sessions and categorizing URLs from the server log files can enhance the data and provide better analysis in student usage.

\section{Web Usage Analysis}

Student usage in online courses is recorded automatically in a form of web access log data at the server level. It is the quantity of the data collected that make the analysis tasks feasible and actionable. Web access $\log$ data, web analysis tools and the proposed XML approach are discussed in the following subsections.

\subsection{Web access log data}

From Apache HTTP Server Project [6], the format of an access log file consists of the IP address of the client (remote host) that made the request to the server, the user ID of the person requesting the document as determined by HTTP authentication, the date and time that the server finished processing the request, the request line from the client is given in double quotes, the status code that the server sends back to the client and the size of the object returned to the client, not including the response headers. Useful information can be derived from the access log files as they capture wide attributes of request data from the web server. They can be used to construct and identify data abstractions, notably user, server sessions, episodes and page views [7], for analyzing navigation patterns. To avoid inconsistency in defining the data abstractions in this paper, Table 1 below summarizes these terms from W3C Web Characterization Activity [8].

Table 1. W3C Definitions (WCA)

\begin{tabular}{ll}
\hline Terms & Definitions \\
\hline User & $\begin{array}{l}\text { The principal using a client to } \\
\text { interactively retrieve and render } \\
\text { resources or resource manifestations. } \\
\text { A delimited set of user clicks across one } \\
\text { or more Web servers. }\end{array}$ \\
Episode & $\begin{array}{l}\text { A subset of related user clicks that occur } \\
\text { within a user session. }\end{array}$ \\
Server & $\begin{array}{l}\text { A collection of user clicks to a single } \\
\text { Session }\end{array}$ \\
Page View & $\begin{array}{l}\text { Visual rendering of a Web page in a } \\
\text { specific client environment at a specific } \\
\text { point in time. }\end{array}$ \\
\hline
\end{tabular}

\subsection{Web analysis tools}

Existing web analysis tools such as WebTrends Log Analyzer and Webalizer use log files to analyze web 
server traffic. They are easy to setup, user friendly and provide customizable template-based reports. Although they are powerful in term of functionalities, the reports are very general and difficult to interpret due to the dynamic structure and content of the web sites. Besides, it is limited to combine with other data sources in the analysis process. These reports do not fulfill the needs of the user for reflecting their usage.

\subsection{Proposed XML approach}

Generating user sessions and categorizing URLs from the server log files can enhance the data and provide better analysis in student usage. To overcome the deficiency from those web analysis tools, an XML model can be used to represent the enriched web log data. XML Tags are self-defined according to the data model Log data are stored in a tree structure format and can be easily transformed and exchanged from one system to another. Table 2 compares the differences between web server log file and XML web log data.

Table 2. Server log and XML log comparison

\begin{tabular}{l|ll}
\hline $\begin{array}{l}\text { Data } \\
\text { Record Series } \\
\text { Extensibility }\end{array}$ & Server Log File & XML Web Log \\
& $\begin{array}{l}\text { Time-based } \\
\text { Limit to server } \\
\text { configuration }\end{array}$ & $\begin{array}{l}\text { User-based } \\
\text { Use XSLT to } \\
\text { transform into } \\
\text { various formats } \\
\text { such as PDF, } \\
\end{array}$ \\
& & HTML, SVG \\
\hline
\end{tabular}

\section{XML Web Log Data Model}

This section describes the implementation of the XML web $\log$ data model and how it is used to represent student usage in online courses. It involves several steps. The first step is the design of an XML schema for the web log data model. The second step is the extracting, transforming and loading (ETL) of data from web server log files into XML conforming to the schema. The third and final step is the use of XSLT style sheets to render the XML data for visualizing and comparing student usage.

\subsection{XML schema design}

An XML schema is designed for implementing the XML web $\log$ data model. It consists of 4 elements, namely Log (root element), User, Session and URL as well as several attributes. Figure 1 below shows the
XML schema of the web log data model and Table 3 summarizes the attributes in each element.

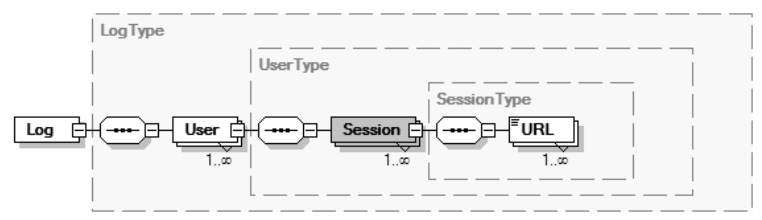

Figure 1. Web log data model

Table 3. Attributes in each element

\begin{tabular}{ll}
\hline Elements & Attributes \\
\hline Log & None \\
User & User ID \\
Session & Session ID, IP Address, Start Time, End Time, \\
& $\begin{array}{l}\text { Duration, Page Count } \\
\text { URL }\end{array}$ \\
\hline
\end{tabular}

\subsection{Extracting, transforming, loading of data}

As discussed in section 3.1, the structure of a web server $\log$ entry contains IP address, user ID, the date time and the URL of the requested page. Table 4 shows a sample log entry. Users are required for authentication in online courses. Their User IDs are captured in the access log files when the web server processes client request. Therefore, user sessions can be identified based on the User IDs and the requested date time attribute in the access log files. Data are imported into a database, mapping the log entry attribute to the corresponding columns with the correct format, such that preprocessing can be performed in an efficient manner. For example, the requested date time attribute is transformed into the DATETIME format when importing into the database. Table 5 shows the structure of the database table storing the access log data. The requested pages can be categorized using SQL by matching the URL path from the structure of the online course. Table 6 shows the relations between page category and URL paths. A script program is used to output the web log data conforming to the XML schema from the temporary database table after extraction and transformation of the raw $\log$ data.

Table 4. A sample log entry

\begin{tabular}{ll}
\hline Attribute & Example \\
\hline IP Address & 203.218 .118 .8 \\
User ID & 50227036 \\
Request Date \& Time & {$[06 /$ Sep/2002:22:51:23 +0800] } \\
Method / URL / Protocol & "GET /a.html HTTP/1.1" \\
Status & 200 \\
Size & 1401 \\
\hline
\end{tabular}


Table 5. Structure of the database table

\begin{tabular}{lll}
\hline Column & Type & Example \\
\hline Session ID & INTEGER & 1 \\
User ID & VARCHAR & 50227036 \\
IP Address & VARCHAR & 203.218 .118 .8 \\
Date Time & DATETIME & 2002-09-06 22:51:23 \\
URL & VARCHAR & /a.html \\
Page & VARCHAR & DOWNLOAD \\
\hline
\end{tabular}

Table 6. Mappings of page and URL paths

\begin{tabular}{ll}
\hline Page & URL \\
\hline Organizer & $\% /$ serve_home?_homepage $\%$ \\
Download & $\% /$ HTML/download/\% \\
Calendar & $\% /$ serve_calendar\% \\
Forum & $\% /$ serve_bulletin $\%$ \\
Schedule & $\% /$ HTML/schedule/\% \\
\hline
\end{tabular}

\subsection{Processing the XML web log data}

There are many ways to analyze student usage from the XML web log data. With the use of XSLT and XPATH, the XML data can be rendered and outputted into various formats such as HTML, SVG, etc. Figure 2 below shows sample XML web log data. Table 7 is the log summary from the use of XSLT in Figure 3.

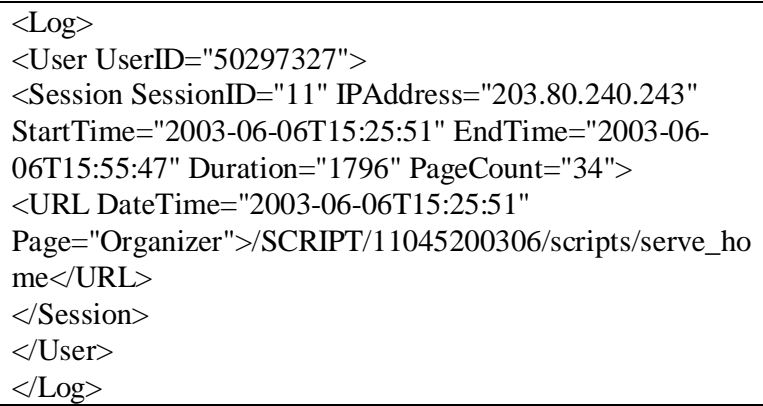

\section{Figure 2. Sample XML web log data}

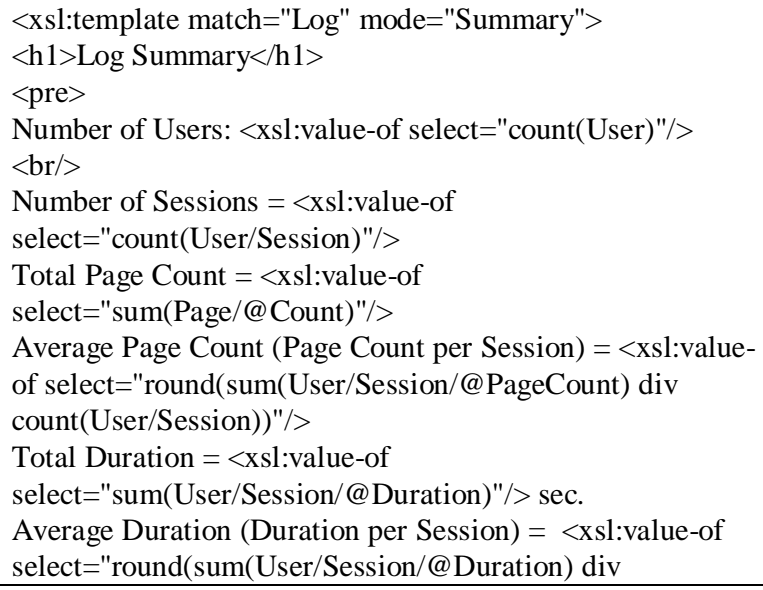

count(User/Session))"/> sec.

$</$ pre $>$

$\langle/$ xsl:template $>$

Figure 3. Sample XSLT for rendering results

Table 7. Log Summary from XSLT in Figure 3

\begin{tabular}{l|l}
\hline Number of Users & 55 \\
Number of Sessions & 1456 \\
Total Page Count & 16765 \\
Average Page Count (Page Count per Session) & 12 \\
Total Duration (sec.) & 285892 \\
Average Duration (Duration per Session) & $196 \mathrm{sec}$. \\
\hline
\end{tabular}

\section{Reflection of Student Usage}

Reflection of student usage is defined as follows: Reflection $=\{$ Recap, Compare $\}$ Recap $=\{$ Extract, Transform, Load $\}$ Compare $=\{$ Reveal, Summarize $\}$

As discussed in section 4, student usage can be recapped by extracting, transforming and loading the web server log files into the XML web data model. The comparison element of reflection involves revealing and summarizing student usage. They are incorporated in the following sub-sections below by presenting several examples of student usage in the online course.

\subsection{Analysis of student access pattern}

Table 8 recaps the user sessions from 2 sample students. Each user session is summarized by the pages accessed. The number after the page indicates the number of visits of each page. The actual URLs can be revealed to reflect the access history. From the result, the student navigated most of the pages in the first user session and only the download pages in other subsequent sessions. This implies that the student uses the online course mostly for download purposes. In fact, most student access patterns are similar to this sample from the result.

\begin{tabular}{cl}
\multicolumn{2}{c}{ Table 8. User sessions from 2 sample } \\
students
\end{tabular}




\begin{tabular}{cl}
4 & Organizer (4), Download (2) \\
5 & Organizer (11), Download (5) \\
6 & Organizer (6), Download (2) \\
7 & Organizer (3), Schedule (1), Information (1) \\
\hline Session & Page Accessed by Student B \\
\hline 1 & Organizer (10), Information (4), Calendar (2), \\
& Schedule (2), Assignments (1) \\
2 & Organizer (4), Download (3) \\
3 & Organizer (4), Download (3) \\
4 & Organizer (4), Download (2) \\
5 & Download (6), Organizer (4) \\
6 & Download (8), Organizer (4) \\
7 & Organizer (4), Download (2)
\end{tabular}

\subsection{Analysis of student usage by page counts}

Page counts can be used for measuring the access frequency of pages. Table 9 below summarizes the percentage of pages accessed by students in the sample online course. The result shows that nearly $50 \%$ and $30 \%$ are related to the organizer pages and the download pages respectively. It indicates that the organizer pages and download pages are most frequently accessed. This implies that most students used to navigate the structure of the course website. The utilization of the download pages is more than that of the other pages such as discussions and quiz, etc.

\subsection{Analysis of student usage by time duration}

Although page counts are useful in analyzing how frequent the pages are accessed, the counts do not differentiate the comprehensiveness or level of details for different pages. This means that a visit to a page with lengthy information and a visit to a page with less detail are treated as equal. Yet, in reality, a student may spend 30 minutes for browsing a page but only 2 minutes for browsing another. For example, the time spent on quiz pages may be relatively longer because the student need time to digest the questions and formulate the answers. In contrast, the time spent on the organizer pages may be relatively shorter. As a supplement, time duration can be used for measuring the time spent by students on some pages. However, time duration is mostly calculated by sessions (not by pages). Programming efforts may be required in order to measure time duration for specific types of pages.

\subsection{Student comparison by page}

Table 9 below also summarizes the percentage of pages accessed by student $A$ and $B$ in the sample online course. The result shows that student A has only accessed the organizer, download and schedule pages while student $B$ has accessed other pages such as discussions, self test, quiz/survey, etc. This implies that student B is a more active student than student A as he/she has contributed more in the online course such as doing self test and participating in the discussions.

Table 9. \% of Page Accessed by Students

\begin{tabular}{lccc}
\hline Page by \% & \multicolumn{3}{c}{ Student } \\
\hline Organizer & All & Student B \\
Download & 48 & 35 & 40 \\
Others & 29 & 63 & 31 \\
Schedule & 7 & 0 & 0 \\
Discussions & 5 & 2 & 2 \\
Information & 3 & 0 & 5 \\
Calendar & 3 & 0 & 2 \\
Assignments & 2 & 0 & 5 \\
Self Test & 1 & 0 & 2 \\
Quiz/Survey & 1 & 0 & 5 \\
Glossary & 1 & 0 & 5 \\
Whiteboard & 0 & 0 & 2 \\
Student Presentations & 0 & 0 & 0 \\
Chat & 0 & 0 & 0 \\
\hline
\end{tabular}

\section{Conclusion}

This article discusses the reflection of student usage in online courses using an XML approach. The implementation of the XML web log data model is described. Using XML to represent $\log$ data is a flexible and extensible method to visualize and compare student usage. Student would be able to recap and compare his/her usage with others and improve in using the online courses. On the other hand, instructor would be able to examine student usage patterns and make refinement to the online course accordingly. Thus, reflecting student usage in online courses is important for both student and instructor.

Since the log data are collected in batch at the end of the semester, one possible improvement is to gather them regularly during the semester, e.g. weekly basis, so that student usage can be reflected continuously. The XML web log data model can be extended to integrate student usage from other online courses for further analysis. 


\section{References}

[1] Lieblein, E., Critical factors for successful delivery of online programs, The Internet and Higher Education, 3(3), 2000, 161-174.

[2] Schifter, C., Teaching in the 21st Century, The Internet and Higher Education, 1(4), 1999, 281-290.

[3] Cheng, C.C., Construction and Evaluation of a WebBased Learning Portfolio System: An Electronic Assessment Tool, Innovations in Education and Teaching International, 38(2), 2001, 144-155.

[4] Dringus, L., \& Terell, S., The framework for directed online learning environments, The Internet and Higher Education, 2(1), 1999, 55-67.
[5] Coldwell, Jo., Mapping Pedagogy to Technology - A Simple Model, In Proceedings of the 2nd International Conference on Advances in Web-Based Learning, 180-192, 2003.

[6] Apache HTTP Server Project. http://www.apache.org

[7] Srivastava, J., Cooley, R., Deshpande, M., Tan, P., Web Usage Mining: Discovery and Applications of Web Usage Patterns from Web Data, ACM SIGKDD, 1 (2), 2000, 12-23

[8] World Wide Web committee web characterization activity. http://www.w3.org/WCA 